\title{
Avaliação da qualidade de vida e tomada de decisão em idosos participantes de grupos socioterápicos da cidade de Arroio do Meio, RS, Brasil
}

Evaluation of the quality of life and decision-making capacity in elderly participating in sociotherapic groups in Arroio do Meio city, Brazil

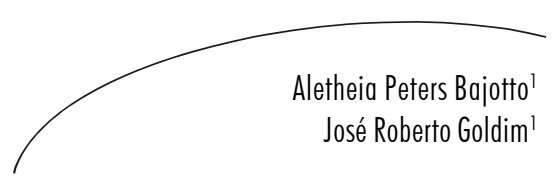

O objetivo do estudo foi avaliar os domínios da qualidade de vida (Questionário WHOQOL-OLD) e a capacidade de tomada de decisão (Questionário de Avaliação do Desenvolvimento Psicológico Moral) de idosos que participam de grupos socioterápicos da cidade de Arroio do Meio, RS. Realizou-se pesquisa caracterizada como transversal, observacional, descritiva, em indivíduos que participavam de grupos de idosos. Da amostra de 133 idosos, o domínio "Intimidade" obteve a média mais expressiva $(69,12+23,52)$; "Autonomia" alcançou uma média baixa $(38,68+16,76)$ e a média do desenvolvimento Psicológico- Moral dos participantes foi de 4,4, situando-os na fase Conscienciosa. Os idosos de Arroio do Meio apresentam-se satisfeitos com a capacidade de estabelecer relacionamentos íntimos e pessoais e com a participação social na comunidade. Apresentaram um escore global de qualidade de vida baixo, mas são completamente capazes de tomar decisões em seu melhor interesse.

\section{Abstract}

The aim of the study was to evaluate the domains of quality of life (WHOQOLOLD Questionnaire) and the ability of decision-making (Questionnaire for Evaluation of the Moral and Psychological Development) of elderly who took part of the socio-therapeutic groups of Arroio do Meio city, Rio Grande do Sul state, Brazil. The research was described as transversal, observative, descriptive, developed among individuals participating in "elderly groups". From the sample formed by 133 elderly, "Intimacy" obtained the most expressive average $(69.12+$ 23.52). "Autonomy" obtained the lowest average $(38.68+16.76)$ and the average

Laboratório de Pesquisa de Bioética e Ética na Ciência, Programa de Pós-Graduação em Gerontologia Biomédica. Pontifícia Universidade Católica do Rio Grande do Sul. Porto Alegre, RS, Brasil.

E-mail: aletheia@bajotto.com.br
Palavras-chave: Idoso. Qualidade de Vida. Tomada de Decisões. Comportamento. 
of "The psycho-moral development" was 4.4, placed in the phase of "Awareness". The elderly from Arroio do Meio are satisfied with the ability of establishing intimate and personal relationships and with social participation in the community. They have low global score on quality of life, but are totally able of taking their own decisions.
Key words: Elderly. Quality of Life. Decision Making. Behavior.

\section{INTRODUÇÃO}

O processo de envelhecimento, seja em sua dimensão física e funcional (geriatria), seja em seus aspectos multidisciplinares (gerontologia), ocorre de forma diferenciada em cada pessoa. Na busca de uma leitura que leve em conta suas múltiplas dimensões, tais como física, mental e social, política, econômica, histórica e cultural, ${ }^{1,2}$ não encontramos na literatura especializada uma definição consensual acerca da velhice.

Até o ano de 2025, o Brasil será o sexto país com o maior número de pessoas idosas no mundo. No país, essa faixa etária é formada por 14,5 milhões de pessoas, representando $8,6 \%$ da população total do país. Esse contundente padrão estatístico de crescimento se torna mais visível quando constatamos que, numa só década, o número de idosos no Brasil cresceu 17\% (em 1991, correspondia a $7,3 \%$ da população).

Além do aumento no número de idosos, também há aumento da expectativa de vida. Estima-se que em 2020, a população com mais de 60 anos no país deva chegar a 30 milhões de pessoas ( $13 \%$ do total), e a expectativa de vida a 70,3 anos. A diferença entre o Brasil, que é considerado um país em desenvolvimento, e os países já desenvolvidos, é que estes últimos foram gradativamente se adaptando e criando políticas que levaram em conta o crescente número de idosos. ${ }^{3}$

A estatística acima encoraja uma reflexão motivada pela preocupação com a garantia aos idosos não apenas de maior longevidade, mas de bem-estar, felicidade, qualidade de vida e satisfação pessoal. ${ }^{4} \mathrm{O}$ foco da presente pesquisa junto aos grupos de idosos, elemento essencial do estudo, se justifica como sendo uma ferramenta em busca das situações cotidianas que possam melhorar a qualidade de vida das pessoas que chegam à terceira idade.

O termo "Grupo socioterápico" define o espaço cuja finalidade é incentivar um resgate prazeroso de atividades sociais, em ambientes exteriores às quatro paredes em que os idosos geralmente vivem uma rotina limitada. Através da formação de um vínculo - proporcionado pela interação dentro dos grupos Socioterápicos - com os elementos do grupo que lhes dê segurança, apoio, compreensão e liberdade, resultados positivos podem ser alcançados, apontando para o desenvolvimento livre e sadio dos componentes do grupo. ${ }^{5}$ Durante os encontros dos grupos de idosos (ou grupos Socioterápicos), os indivíduos têm oportunidade de dividir experiências, promover eventos em um espaço onde todos buscam algo em comum: interação social entre a comunidade idosa de uma determinada região.

O presente artigo objetiva apresentar os resultados da avaliação da qualidade de vida e a capacidade de tomada de decisão em pessoas acima de 60 anos de idade, moradoras da cidade de Arroio do Meio, RS, participantes de grupos socioterápicos ou grupo de idosos. Pesquisar os domínios que contribuem para maior qualidade de vida do idoso pode oferecer uma possibilidade de desmistificar algumas falsas dicotomias, como as que colocam a saúde como determinante para a qualidade de vida nessa faixa etária ou a ideia de que o idoso é vulnerável e, por consequência, incapaz de autogovernar-se.

Uma mudança demográfica contundente como essa formula aos especialistas, às pessoas públicas e à coletividade, um dos maiores desafios sociais da história e uma expressiva demanda por estudos e análises para uma melhor definição de políticas públicas de saúde no envelhecimento. Isto porque os avanços científicos e técnicos acenam ao ser humano com a possibilidade factível do 
alcance dos 110 ou mesmo 120 anos de vida uma expectativa que corresponderia aos limites biológicos - ainda neste século. ${ }^{2}$

\section{METODOLOGIA}

A pesquisa foi do tipo transversal, observacional descritiva, realizada em indivíduos de ambos os sexos, com idade igual ou superior a 60 anos. A avaliação dos pacientes foi realizada na cidade de Arroio do Meio, RS, com o conhecimento da Secretaria Municipal da Saúde e apoio do Conselho Municipal do Idoso. A aplicação dos instrumentos de avaliação foi realizada durante o encontro dos "Grupos de Idosos", de forma autoaplicada assistida, sendo a coleta de dados realizada entre outubro e novembro de 2007 e de fevereiro a maio de 2008.
Foram utilizados dois instrumentos para avaliação dos idosos: WHOQOL-OLD e Instrumento de Avaliação do Desenvolvimento Psicológico-Moral. O World Health Organization Quality of Life Group (Grupo WHOQOL) desenvolveu uma escala dentro de uma perspectiva transcultural para medir qualidade de vida em adultos. Considerou como características fundamentais o caráter subjetivo da qualidade de vida (englobando aspectos positivos e negativos) e sua natureza multidimensional. ${ }^{6,7,8}$

O Instrumento de Avaliação do Desenvolvimento Psicológico-Moral é composto por um conjunto de frases de fácil compreensão que enquadram os sujeitos numa das sete fases do desenvolvimento psicológicomoral. ${ }^{9}$ (Quadro 1)

Quadro1 - Fases e características do desenvolvimento psicológico-moral.

\begin{tabular}{|l|c|l|}
\hline $\begin{array}{c}\text { Fases do } \\
\text { desenvolvimento } \\
\text { psicológico-moral }\end{array}$ & Pontuação & \multicolumn{1}{c|}{ Características } \\
\hline 1 - Pré-social & $0,1-1$ & Início do desenvolvimento, ainda pré -verbal. \\
\hline 2 - Impulsiva & $1,1-2$ & $\begin{array}{l}\text { Decisões tomadas por impulso, baseado apenas } \\
\text { em seu desejo, não considerando as } \\
\text { informações. }\end{array}$ \\
\hline 3 - Oportunista & $2,1-3$ & $\begin{array}{l}\text { Supervalorização dos desejos e valorização das } \\
\text { informações para atingi-los. }\end{array}$ \\
\hline 4 - Conformista & $3,1-4$ & $\begin{array}{l}\text { Crenças do indivíduo se sobrepõem ao seu } \\
\text { próprio desejo e justificam as contingências } \\
\text { impostas pelo meio. }\end{array}$ \\
\hline 5 - Conscienciosa & $4,1-5$ & $\begin{array}{l}\text { Capacidade de to mar decisões de forma mais } \\
\text { autônoma, cotejando os desejos e as crenças, } \\
\text { mas ainda passível de constrangimento, pois } \\
\text { não tem a noção de regra introjetada. }\end{array}$ \\
\hline 6 - Autônoma & $5,1-6$ & $\begin{array}{l}\text { Possui as regras introjetadas e é capaz de tomar } \\
\text { decisões livres de constrangime nto de forma } \\
\text { autônoma. }\end{array}$ \\
\hline 7 - Integrada & $6,1-7$ & $\begin{array}{l}\text { Possui a noção de regra individual introjetada, } \\
\text { mas se vê como parte de um todo, com a } \\
\text { compreensão da independência existente. }\end{array}$ \\
\hline
\end{tabular}

Fonte: Souza, $1968 .^{35}$ 
O estudo obteve aprovação do Comitê de Bioética da PUCRS (CEP 07/03799) e cumpriu com os princípios éticos contidos na Declaração de Helsinki, além de atender à legislação pertinente. Todos os participantes do estudo receberam explicações claras e concisas em relação à pesquisa, procedendo à assinatura do termo de consentimento livre e esclarecido.

\section{RESULTADOS}

Participaram da pesquisa 133 idosos, com média de 69,02 +6,50 anos e idades entre 60 e 89 anos. Destes, $81(60,9 \%)$ eram do sexo feminino e $52(39,1 \%)$ do sexo masculino.
Em relação à escolaridade, 95 idosos possuem o $1^{\mathrm{o}}$ grau incompleto $(71,42 \%), 31$ idosos completaram o $1^{\circ}$ grau $(23,30 \%)$. Apenas um participante possui o $2^{\circ}$ grau completo $(0,07 \%)$, três idosos têm o $2^{\circ}$ grau incompleto $(2,25 \%)$ e três são analfabetos (2,25\%). Dentre os participantes do estudo, 66 eram aposentados (49,62\%), 47 agricultores (35,38\%), 13 idosas classificam-se como “do lar” (9,77\%), seis são domésticas (4,51\%) e um idoso é motorista (0,07\%).

Avaliação dos domínios do WHOQOL-OLD

Foram obtidos os escores médios e desvios padrão dos seis domínios do WHOQOL-OLD, os quais são apresentados abaixo no quadro 2 . Dos 133 participantes da amostra, foi possível utilizar os dados de 132 participantes.

Quadro 2 - Escores médios e variabilidade dos seis domínios do WHOQOL-OLD em uma amostra de 132 idosos de Arroio do Meio/Brasil.

\begin{tabular}{|l|c|c|c|}
\hline \multicolumn{1}{|c|}{$\begin{array}{c}\text { Faceta/Domínio do } \\
\text { WHOQOL-OLD }\end{array}$} & Média & Desvio Padrão & N \\
\hline $\begin{array}{l}\text { Funcionamento dos } \\
\text { Sentidos (SAB) }\end{array}$ & 42,14 & 11,85 & 132 \\
\hline Autonomia (AUT) & 38,68 & 16,76 & 132 \\
\hline $\begin{array}{l}\text { Atividades assadas, } \\
\text { Presentes e Futuras (PPF) }\end{array}$ & 49,00 & 16,77 & 132 \\
\hline Participação Social (SOP) & 68,93 & 18,06 & 132 \\
\hline Morte e Morrer (DAD) & 27,88 & 17,24 & 132 \\
\hline Intimidade (INT) & 69.12 & 23,52 & 132 \\
\hline Total & 49,29 & 6,88 & \\
\hline
\end{tabular}

Fonte: Autora, Arroio do Meio RS, 2008.

Os valores obtidos indicam proporcionalmente a qualidade de vida - valores mais altos indicam alta qualidade de vida associada ao domínio e valores mais baixos o oposto. No domínio "Morte e Morrer", no entanto, ocorre o inverso.

A avaliação global obtida desta amostra (49,29 $+6,88)$ alcançou um valor próximo ao nível médio e inferior ao obtido na amostra de idosos do RS $(68,55+9,80)$.
O primeiro domínio do questionário trata do Funcionamento dos Sentidos. Avalia o impacto da perda do funcionamento dos sentidos (perdas na audição, visão, paladar, olfato, tato) na qualidade de vida. O valor encontrado foi 42,14 $+11,85$, ficando abaixo do valor central da distribuição.

O domínio Autonomia avalia a independência do idoso para tomar suas próprias decisões, sentir que controla seu futuro, fazer as coisas que 
gostaria de fazer ou acreditar que as pessoas ao seu redor respeitam sua liberdade. $\mathrm{O}$ valor $\mathrm{da}$ amostra foi de 38,68 + 16,76, um score abaixo do nível médio.

Nas Atividades passadas, presentes e futuras, avaliou-se a satisfação com as realizações na vida, das oportunidades de novas realizações, reconhecimento merecido na vida, da felicidade com o que ainda se espera do futuro e com objetivos a serem alcançados. $O$ valor obtido na amostra dos idosos de Arroio do Meio foi de 49 +16,77 -próximo do valor central da distribuição.

O domínio Participação Social demonstrou a satisfação pessoal com as atividades diárias, com o uso do tempo, com o nível pessoal de atividade e com as oportunidades de participar nas atividades da comunidade. O valor obtido com a amostra foi de $68,93+18,06$ e ficou acima do nível médio.
O domínio Morte e Morrer avalia as preocupações e os medos acerca da morte e do morrer. O escore foi de 27,88 +17,24 - valor abaixo do nível médio e inferior ao obtido na amostra de idosos do RS $(32,07+24,37)$.

O domínio Intimidade avalia a capacidade de estabelecer relacionamentos íntimos e pessoais e apresentou um valor de 69,12+23,52. Este se encontra acima do nível médio e semelhante ao obtido na amostra de idosos do RS $(69,00+15,81)$.

\section{Avaliações do desenvolvimento psicológico- moral da amostra}

$\mathrm{Na}$ amostra de 133 idosos, 122 questionários encontravam-se completos e foram analisados. A fase Pré-Social, como esperado, não teve qualquer idoso ali classificado, pois não ocorre em indivíduos adultos e idosos (quadro 3).

Quadro 3 - Distribuição dos 123 participantes da amostra quanto às fases de desenvolvimento psicológico-moral.

\begin{tabular}{|l|c|c|}
\hline $\begin{array}{l}\text { Fases do desenvolvimento } \\
\text { Psicológico-Moral }\end{array}$ & Fase & $\mathrm{N}$ \\
\hline Pré-social & 1 & - \\
\hline Impulsiva & 2 & $3,2 \%(4)$ \\
\hline Oportunista & 3 & $5,7 \%(7)$ \\
\hline Conformista & 4 & $17,9 \%(22)$ \\
\hline Conscienciosa & 5 & $38,2 \%(47)$ \\
\hline Autônoma & 6 & $32,5 \%(40)$ \\
\hline Integrada & 7 & $2,4 \%(3)$ \\
\hline Total & & $100 \%(123)$ \\
\hline
\end{tabular}

Fonte: Autora, Arroio do Meio RS 2008.

Quatro participantes encontraram-se no nível 2; sete idosos encaixaram-se no nível 3; o quarto nível do desenvolvimento abrigou 22 idosos (terceiro lugar); o quinto nível (a fase Conscienciosa) teve o maior número de idosos, 47; o sexto nível contou com 40 idosos (segundo lugar) e três participantes atingiram o nível integrado.
Os dados obtidos permitem afirmar que apenas $8,9 \%$ dos idosos não apresentavam desenvolvimento psicológico-moral que permita tomar decisões no seu melhor interesse. Os demais 112 idosos, isto é, 91,2\% da amostra, tinham plenas condições de decidir de forma adequada. Vale destacar que três idosos atingiram o nível integrado, considerado máximo no desenvolvimento psicológico-moral de uma pessoa. 


\section{DISCUSSÃO}

Após a análise dos resultados, o primeiro ponto a ser realçado é a prevalência do sexo feminino no estudo (60,9\%), corroborando a literatura existente. As mulheres são a maioria da população e vivem mais do que os homens em quase todos os lugares. Este fato se reflete na maior taxa de mulheres por homens em grupos etários mais velhos. Na Europa, em 2002, havia 678 homens para cada 1.000 mulheres com 60 anos ou mais. Em regiões menos desenvolvidas, havia 879 homens para cada 1.000 mulheres. ${ }^{10}$

As mulheres correspondem aproximadamente a dois terços da população acima de 75 anos em países como Brasil e África do Sul, tendo a vantagem da longevidade, mas sendo vítimas mais frequentes da violência doméstica e de discriminação no acesso à educação, salário, alimentação, trabalho significativo, assistência à saúde, heranças, medidas de seguro social e poder político. ${ }^{1,11}$

A baixa escolaridade dos participantes da pesquisa $\left(71,4 \%\right.$ possuem $1^{\circ}$ grau incompleto) também aparece em outros estudos, ${ }^{12}$ sendo que quanto mais avançada a idade, maior será a proporção de pessoas com baixa escolaridade. ${ }^{13}$

O escore obtido no domínio autonomia na amostra de Arroio do Meio foi inferior aos resultados da amostra de Erechim/RS (quadro 4). Este dado aparentemente paradoxal, quando comparado ao verificado no desenvolvimento psicológico-moral, demonstra que, apesar de possuir condições para tomar decisão, de fato não exercem essa capacidade.

Quadro 4 - Comparação entre os domínios do WHOQOL-OLD, respectivamente, da amostra dos idosos de Erechim ( $n=670)$ e dos idosos de Arroio do Meio ( $n=132)$, RS.

\begin{tabular}{|l|c|c|c|c|}
\hline \multicolumn{1}{|c|}{ Faceta/Domínio do WHOQOL-OLD } & \multicolumn{2}{c|}{ Média } & \multicolumn{2}{c|}{ Desvio-padrão } \\
\hline Funcionamento dos Sentidos (SAB) & 67,6 & 42,14 & 20,80 & 11,85 \\
\hline Autonomia (AUT) & 66,4 & 38,68 & 13,14 & 16,76 \\
\hline Atividades passadas, Presentes e Futuras (PPF) & 69,3 & 49,00 & 11,3 & 16,77 \\
\hline Participação Social (SOP) & 72,10 & 68,93 & 10,70 & 18,06 \\
\hline Morte e Morrer (DAD) & 33,00 & 27,88 & 24,40 & 17,24 \\
\hline Intimidade (INT) & 69,00 & 69,12 & 16,80 & 23,52 \\
\hline Total & 68,55 & 49,29 & 9,80 & 6,88 \\
\hline
\end{tabular}

Fonte: Celich, Erechim RS, $2008{ }^{37}$ e dados da autora, Arroio do Meio RS, 2008.

A autonomia pessoal, ou seja, a habilidade de fazer escolhas livres que um ser humano exerce em sua vida, é considerada crucial para a qualidade de vida de uma pessoa de idade avançada. ${ }^{14} \mathrm{O}$ princípio da autonomia, denominação mais comum pelo qual é conhecido o princípio do respeito às pessoas, exige aceitação que elas se autogovernem e sejam autônomas, tanto em suas escolhas quanto em seus atos. ${ }^{15,16}$

O valor do funcionamento dos sentidos desta pesquisa indica que os idosos têm uma percepção de perda na audição, visão, paladar, olfato, tato, pois os índices obtidos foram inferiores à média e inferiores aos obtidos em uma amostra de 670 idosos na cidade de Erechim $(67,60+20,08) .{ }^{17}$

As atividades passadas, presentes e futuras obtiveram escore menor que da amostra dos idosos de Erechim, o que denota certa insatisfação em relação às conquistas da vida em comparação com aquilo que se anseia. A participação social dessa amostra obteve o segundo maior escore, juntamente com o segundo maior desvio padrão, atrás apenas de intimidade. Outros estudos vêm observando que os contatos sociais são um componente tão valioso para a boa qualidade de vida quanto a saúde. ${ }^{18,19,20}$ 
Dentre inúmeros fatores, as pesquisas têm demonstrado grande necessidade, das pessoas desta faixa etária, de atenção e interesse das pessoas que as cercam..$^{21} \mathrm{~A}$ participação social dos idosos em seus grupos parece ser um fator que traz atenção e a sensação de pertencimento à sociedade.

Os momentos de felicidade dos idosos estão relacionados ao contato familiar e social, à autonomia e à capacidade para trabalhar. Esse quadro constitui um conjunto de fatores que traz valor pessoal, autoestima e bem-estar, chamando atenção para o fato de que, mais uma vez, o domínio saúde não aparece como determinante na qualidade de vida com sucesso. ${ }^{22,23}$

O "grupo Socioterápico" parece cumprir o papel de incentivar um resgate prazeroso de atividades sociais, formando um vínculo com os elementos do grupo e lhes proporcionando segurança, apoio, compreensão e liberdade.

A morte e o morrer apresentaram, juntos, os menores escores da pesquisa, o que demonstra a pouca preocupação e a coragem dos idosos quando o assunto é a morte. $\mathrm{Na}$ cidade, os moradores gozam de atendimento médico gratuito e de boa qualidade, o que pode explicar tal postura diante da realidade da morte. Outro aspecto a considerar, quando o assunto é morte e morrer, é a espiritualidade. Percebe-se grande adesão à religião por parte da população. Também nos grupos de Idosos, a espiritualidade se faz presente quando no início de cada encontro, a abertura é feita com uma oração, o que demonstra o grande apego à espiritualidade.

A literatura científica já demonstra interesse pela questão da religião, da fé, da espiritualidade, uma vez que estas apresentam relação com a cura de doenças e qualidade de vida da pessoa. ${ }^{19,24,25} \mathrm{~A}$ própria descendência alemã já traz, em seus indivíduos mais longevos, uma história de muitas perdas, guerras, e isto pode contribuir para que estes idosos com idade mais avançada não temam a morte.

O domínio intimidade, que investiga companheirismo e amor, apresentou o maior escore da pesquisa, o que nos leva a concluir que os idosos de Arroio do Meio têm um envelhecimento pleno de satisfação com seus companheiros e aqueles que os rodeiam.

As estruturas familiares permitem que de três a quatro gerações da mesma família continuem vivendo sob o mesmo teto, o que afasta a possibilidade de solidão que frequentemente os idosos experimentam longe da família. As atividades de trabalho pesado são assumidas pelos filhos quando estes atingem idade para tanto. Assim, na terceira idade, o idoso já pode contar com mão de obra de filhos e netos na roça, restando ao idoso, quando necessária sua ajuda, as lidas da casa e o cuidado dos netos e bisnetos.

Uma vez que os idosos permanecem no seio familiar durante a idade avançada, afasta-se o sentimento da "síndrome do ninho vazio" (conhecido como o processo pelo qual os pais passam quando os filhos vão embora de casa). ${ }^{26,27}$

\section{CONCLUSÃO}

O baixo valor do escore geral do WHOQOLOLD encontrado na pesquisa parece não ser determinante para o impedimento da tomada de decisão, expresso por uma média alta.

O escore mais alto de todos os domínios do WHOQOL-OLD foi da intimidade, seguido pelas atividades sociais. Assim sendo, o suporte familiar e a rede social representam felicidade e satisfação para essa população. $\mathrm{O}$ elo que une comunidades como essa que foi alvo do estudo é muito forte e parece contribuir para que mesmo na idade mais avançada, os indivíduos sejam respeitados e sintam-se como parte da sociedade.

Saúde, primariamente vista como essencial para uma boa qualidade de vida nas idades avançadas não aparece nesta pesquisa como decisivo para a qualidade de vida dessa população. Os idosos de Arroio do Meio não apresentam escore global de qualidade de vida significativo; por outro lado, apresentam capacidade para gerir suas decisões, quando a maior parte da amostra 
se encontra na fase Conscienciosa do desenvolvimento psicológico-moral. Devemos considerar as limitações deste estudo, pois estamos abordando uma população de uma região específica, e embora o número de idosos entrevistado seja significativo, não representa a totalidade de idosos daquela comunidade.

Mesmo diante de algumas limitações, na busca em proporcionar um envelhecimento ativo aos nossos indivíduos de cabelos brancos, um dos caminhos é incentivar sua participação na sociedade - valorizando, por exemplo, os Grupos Socioterápicos. Investimentos maciços em atividades que promovam o resgate prazeroso do idoso na sociedade podem fazer a diferença, em conjunto com outros fatores, para que a terceira idade não seja vista como o final da trajetória, mas sim, um recomeço. Proporcionar o convívio do idoso no seio da família, respeitar o direito de gerir suas decisões também são pontos-chave para transformar essa revolução demográfica não em caos, mas numa conquista para a humanidade.

\section{REFERÊNCIAS}

1. Organização Mundial da Saúde (OMS).

Envelhecimento ativo: uma política de saúde.

Brasília: Organização Pan-Americana da Saúde; 2005.

2. Veras RP, Caldas PC. Promovendo a saúde e a cidadania do idoso: o movimento das universidades da terceira idade. Cienc Saude Colet. 2004;9(2):423-32.

3. Instituto Brasileiro de Geografia e Estatística.Perfil dos Idosos Responsáveis pelos Domicílios. [ Acesso em 17 out 2009].Disponível em: URL: http:// www.ibge.gov.br/home/presidencia/noticias/ 25072002pidoso.shtm

4. Albuquerque AS, Tróccoli BT. Desenvolvimento de uma escala de bem-estar subjetivo. Psicologia: teoria e pesquisa. 2004;20:153-64.

5. Zimerman G. Velhice, aspectos biopsicossociais. Porto Alegre: Artmed; 2000. 228p.

6. Fleck MPA, Chachamovich E, Trentini CM. Projeto WHOQOL-OLD: método e resultados de grupos focais no Brasil. Rev de Saude Publica, 2003;37(6):793-9.

7. Power M, Schmidt S. Manual WHOQOL-OLD. In: Chachamovich E, Fleck MPA. Brasília: OMS; 2006.

8. Power M, Schmidt S. Manual WHOQOL-OLD [ Acesso em 15 jun 2009].Disponível em: http:// ufrgs.br/psiq/WHOQOLOLD\%Manual\%20POrtugues.pdf

9. Goldim JR. O consentimento informado e a adequação de seu uso na pesquisa em seres humanos .Tese [ Doutorado em Ciências Médicas]-UFRGS; 1999.

10. Farenzema WP. Qualidade de vida em grupo de idosos de Veranópolis . Porto Alegre.Dissertação [ Mestrado em Ciências da Saúde]-Universidade Pontifícia Católica; 2007.

11. Mastroeni MF ,et al. Perfil demográfico de idosos da cidade de Joinville: estudo de base domiciliar. Rev Bras Epidemiol. 2007;10(2):190-201.

12. Celich KLS. Domínios de qualidade de vida e capacidade para a tomada de decisão em idosos participantes de grupos da terceira idade. Porto Alegre. Dissertação [ Mestrado em Geriatria e Gerontologia]-Universidade Pontifícia Católica; 2008.

13. Timm LA. Qualidade de vida no idoso e sua relação com o Lócus de Controle. Porto Alegre.Dissertação [Mestrado em Psicologia]Universidade Pontifícia Católica ; 2006.

14. Matsui M, Capezuti E. Perceived autonomy and self-care resources among senior center users. Nurs. 2008;29(2):141-7

15. Clotet J. Bioética uma aproximação. Porto Alegre: EDIPUCRS; 2003.112p.

16. Goldim JR. Bioética e envelhecimento. In: Freitas EV, Py L, Néri AL, Cançado FAX, Gorzoni ML, Rocha SM. Tratado de geriatria e gerontologia. Rio de Janeiro: Guanabara Koogan; 2002. p. 85-99.

17. Cabrero G. Participación Social de las personas mayores. Madrid: IMSERSO; 1997.

18. Joia LC, Ruiz T, Donalisio MR. Life satisfaction among elderly population in the city of Botucatu. 2008. [Acesso em 12 out 2010]. Disponível em: http:// www.scielo.br/scielo.php?script $=$ sci_arttext \&pid=S0034 89102007000100 018\&lng =en\&nrm = iso.doi:10.1590/S003489102007000100018 
19. Farquhar M. Elderly people's definitions of quality of life. Soc Sci Med. 1995;41:1439-46.

20. Vecchia RD ,et al. Qualidade de vida na terceira idade: um conceito subjetivo. Rev Bras Epidemiol. 2005; 8(3):246-52.

21. Van den Boer S, Van den Berg JM. Towards a more humane care for the elderly: ethical reflection. Tijdschr Gerontol Geriatr. 1997;28(5):204-8.

22. Luz MMC, Amatuzzi MM. Vivências de felicidade de pessoas idosas. Estud Psicol. 2008;25(2):303-7

23. Valentine N, Darby C, Bonsel GJ. Which aspects of non-clinical quality of care are most important? Results from WHO's general population surveys of "health systems responsiveness" in 41 countries. Soc Sci Med. 2008;66(9):1939-50.

24. Saad M, Masiero D, Batisttella LR. Espiritualidade baseada em evidências. Acta Fis. 2001;8(3): 107-112

25. Panzini RG, et al. Espiritualidade/religiosidade e qualidade de vida. In: Fleck MP . A avaliação da qualidade de vida: guia para profissionais da saúde. Porto Alegre: Artmed; 2008. p. 178-96.

26. Leite MT ,et al. Idosos residentes do meio urbano e sua rede de suporte familiar e social. Texto Contexto Enf. 2008;17(2):250-7.

27. Liu LJ, Guo Q. Life satisfaction in a sample of empty-nest elderly: a survey in the rural area of a mountainous county in China. Qual Life Res. 2008;17(6):823-30. 
